\title{
Clinical Evaluation of the Effectiveness of Hyaluronic Acid Topical Application on Free Gingival Graft Healing and Dimensional Changes
}

\author{
Auday Al Basher ${ }^{1}$, Tareq Alshwiki ${ }^{2}$, Tarek Kasem ${ }^{3}$
}

\begin{abstract}
Aim and objective: This study aims to evaluate the use of hyaluronic acid (HA) on the free gingival graft (FGG) applied to mandibular canines with Miller class I or II gingival recession (GR) or without attached gingiva by evaluating tissue healing, swelling, and dimensional changes. Materials and methods: This split-mouth clinical study has an examiner-patient blinded design. Eighteen patients with bilateral class I or II of Miller classification of GR in the mandibular canine region with $<2 \mathrm{~mm}$ of attached gingiva were treated with FGG and randomly assigned to experience either natural healing (control group) or HA-assisted healing (test group). Tissue healing index, swelling, and dimensional changes were registered at baseline, 1, and 3 months post-surgery. Statistical analysis was performed to examine differences between study groups.

Results: The test group showed better healing and swelling compared to the control group and these differences were statistically significant. No statistically significant difference was found for dimensional changes between groups.

Conclusion: It may be concluded that within the limits of this study that the adjunctive use of HA may be beneficial in the healing process of FGG. Clinical significance: The application of HA helped improving the healing of FGG, which makes the process faster and more predictable. Keywords: Free gingival graft, Gingival recession, Hyaluronic acid, Mucogingival surgery.

World Journal of Dentistry (2020): 10.5005/jp-journals-10015-1763
\end{abstract}

\section{INTRODUCTION}

Gingival recession (GR) is described as an apical displacement of the gingival margin beneath the cementoenamel junction (CEJ). ${ }^{1,2}$ The exposure of the root causes major clinical problems including esthetic, hypersensitivity, and periodontal attachment loss. ${ }^{3}$

The free gingival graft (FGG) is considered as one of the most used mucogingival surgical procedures for the treatment and management of mucogingival deformities around natural teeth and implants. ${ }^{4,5}$

One of the biggest disadvantages of FGG procedure is postoperative graft shrinkage. ${ }^{6}$ This shrinkage is related to several factors including graft thickness, ${ }^{7}$ gingival tissue phenotype, and surgical procedure. ${ }^{8}$

Factors affecting the development of a new blood supply between the graft and the recipient site play an essential role in graft shrinkage. ${ }^{7}$

Hyaluronic acid (HA) exists in most of the body fluids, such as saliva and crevicular gingival fluid, and is a component of gingival tissue and the cellular membrane of some cells, like fibroblasts, chondrocytes, and lymphocytes type B. ${ }^{9,10}$ Hyaluronic acid takes part in the formation of periodontal ligament and by a less extent in alveolar bone formation. ${ }^{11}$

Hyaluronic acid helps in stimulating the inflammatory response in phagocytes, endothelial cells, basophils, and some of the epithelial cells as well as contributing in wound healing and scar formation., ${ }^{9,12}$

Considering the composition of $\mathrm{HA}$ and its role, it has been used in the medical and the esthetical fields. In dentistry, HA has been used for several purposes and treatments, such as wound healing after tooth extraction, ${ }^{13,14}$ degenerative joint disorder of
${ }^{1-3}$ Department of Periodontology, Faculty of Dentistry, Damascus University, Damascus, Syria

Corresponding Author:Tareq Alshwiki, Department of Periodontology, Faculty of Dentistry, Damascus University, Damascus, Syria, Phone: +963967896826, e-mail: Kaseem.tareq@yahoo.com

How to cite this article: Al Basher A, Alshwiki T, Kasem T. Clinical Evaluation of the Effectiveness of Hyaluronic Acid Topical Application on Free Gingival Graft Healing and Dimensional Changes. World J Dent 2020;11(5):361-366.

Source of support: Nil

Conflict of interest: None

the temporomandibular joint, ${ }^{15}$ sinus lifting instead of applying any graft, and oral ulcers management. ${ }^{16,17}$

In periodontology, $\mathrm{HA}$ is used as an adjunctive material for surgical or non-surgical periodontal treatment to reduce inflammation and improve healing. ${ }^{18,19}$

This study aims to evaluate the use of HA on the FGG applied to mandibular canines with Miller class I or II GR or without attached gingiva by evaluating tissue healing, swelling, and dimensional changes.

\section{Materials and Methods}

The study was conducted in the Department of Periodontology, Damascus University. The study protocol was approved by the Ethics Committee at the Faculty of Dentistry. All participated patients were informed of the study protocol and their written consent was obtained.

(O) The Author(s). 2020 Open Access This article is distributed under the terms of the Creative Commons Attribution 4.0 International License (https://creativecommons. org/licenses/by-nc/4.0/), which permits unrestricted use, distribution, and non-commercial reproduction in any medium, provided you give appropriate credit to the original author(s) and the source, provide a link to the Creative Commons license, and indicate if changes were made. The Creative Commons Public Domain Dedication waiver (http://creativecommons.org/publicdomain/zero/1.0/) applies to the data made available in this article, unless otherwise stated. 


\section{Study Sample}

This split-mouth clinical trial had an examiner-patient blinded design. Eighteen patients with bilateral class I or II Miller GR in the mandibular canine region and $<2 \mathrm{~mm}$ of attached gingiva were assigned to be treated with FGG sides were allocated randomly through a coin toss for the first case and then rotated in the remaining cases either to receive a standard FGG procedure (control group) (Fig. 1) or FGG with HA application (test group) (Fig. 2). The study subjects were recruited between 2018 and 2019 according to the following criteria.

\section{Inclusion Criteria}

The patients were older than 20 years and presenting bilateral class I or II Miller GR in the mandibular canine region. The included teeth were vital and had no caries or restorations. The plaque and the gingival index scores were $<1$. All patients had to be systematically healthy and non-smokers.

\section{Exclusion Criteria}

Patients with the following conditions were excluded:

- Periodontal probing depth higher than $3 \mathrm{~mm}$.

- Patient receiving medical therapy that interferes with wound healing process.
- Pregnant and lactating woman.

- Patient with previous surgical procedure in the study area and patients undergoing orthodontic therapy.

\section{Presurgical Procedure}

All patients received basic scaling, plaque control, and oral hygiene instructions. However, only patients with complete plaque control were assigned for surgery.

\section{Surgical Procedure}

All surgical procedures were performed by one qualified resident of the Department of Periodontology at the Faculty of Dentistry, Damascus University ( $A B)$ according to the surgical technique described by Langer and Langer. Both sides were treated in the same surgical session.

Local anesthesia was achieved with $2 \%$ lidocaine with 1:100,000 epinephrine. A horizontal incision was made at the mucogingival line by \#15 surgical blade. The incision included the mesial and the distal papilla adjacent to the recession area. A split thickness flap was performed to keep the periosteum covering the bone in the recipient site intact and the coronal tissues to the horizontal incision were de-epithelized. The denuded root surface was treated with a curette and rinsed with saline. A saline-moistened gauze was
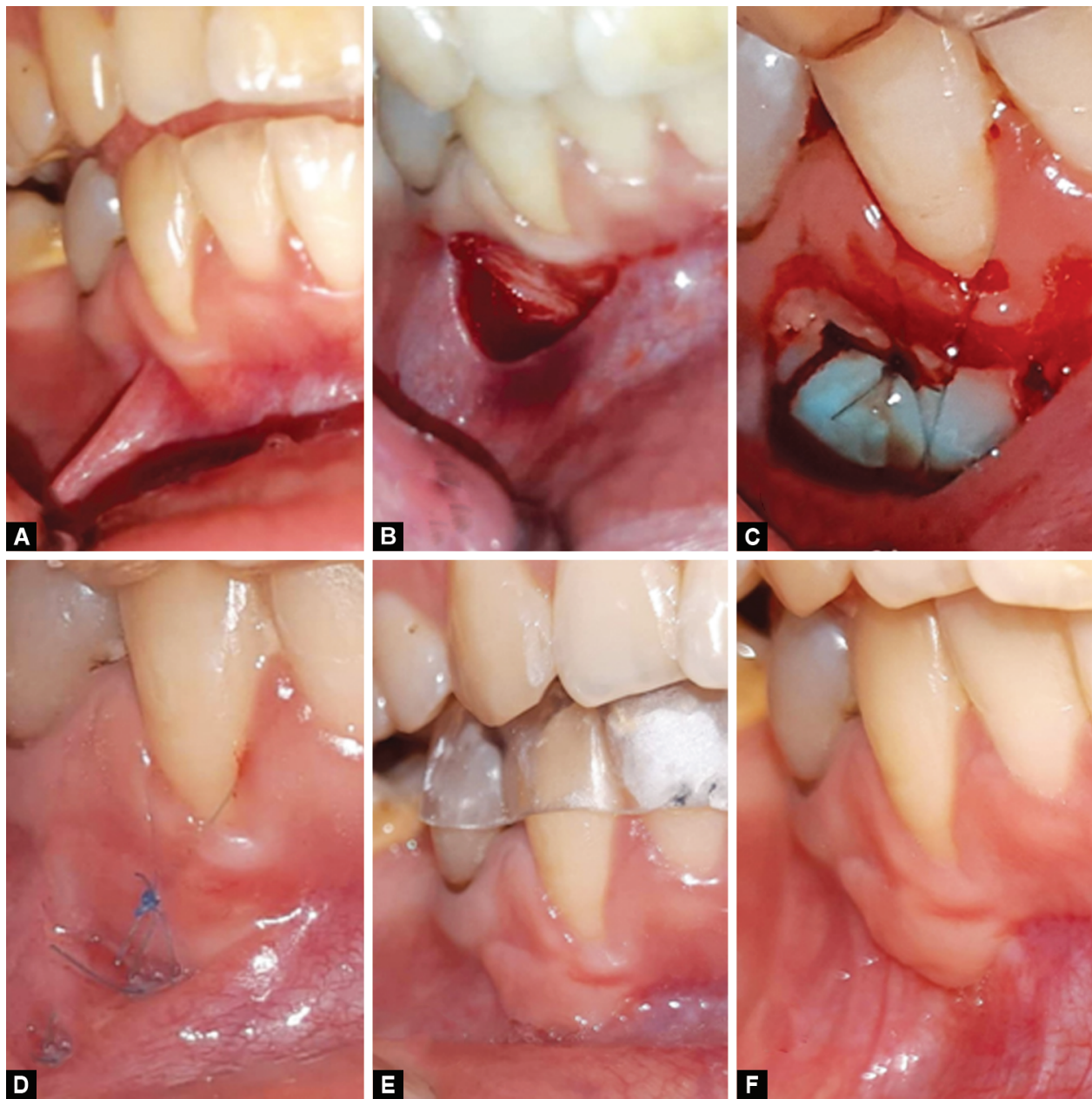

Figs 1 A to F: Free gingival graft (FGG) side: (A) Gingival recession Miller's class II on tooth 33 for the same patient in Figure 2; (B) Recipient bed prepared; (C) Adaptation of the FGG at the recipient area, suture tide; (D) Graft healing at 14th day; (E) 1 month; (F) 3 months post-surgery 

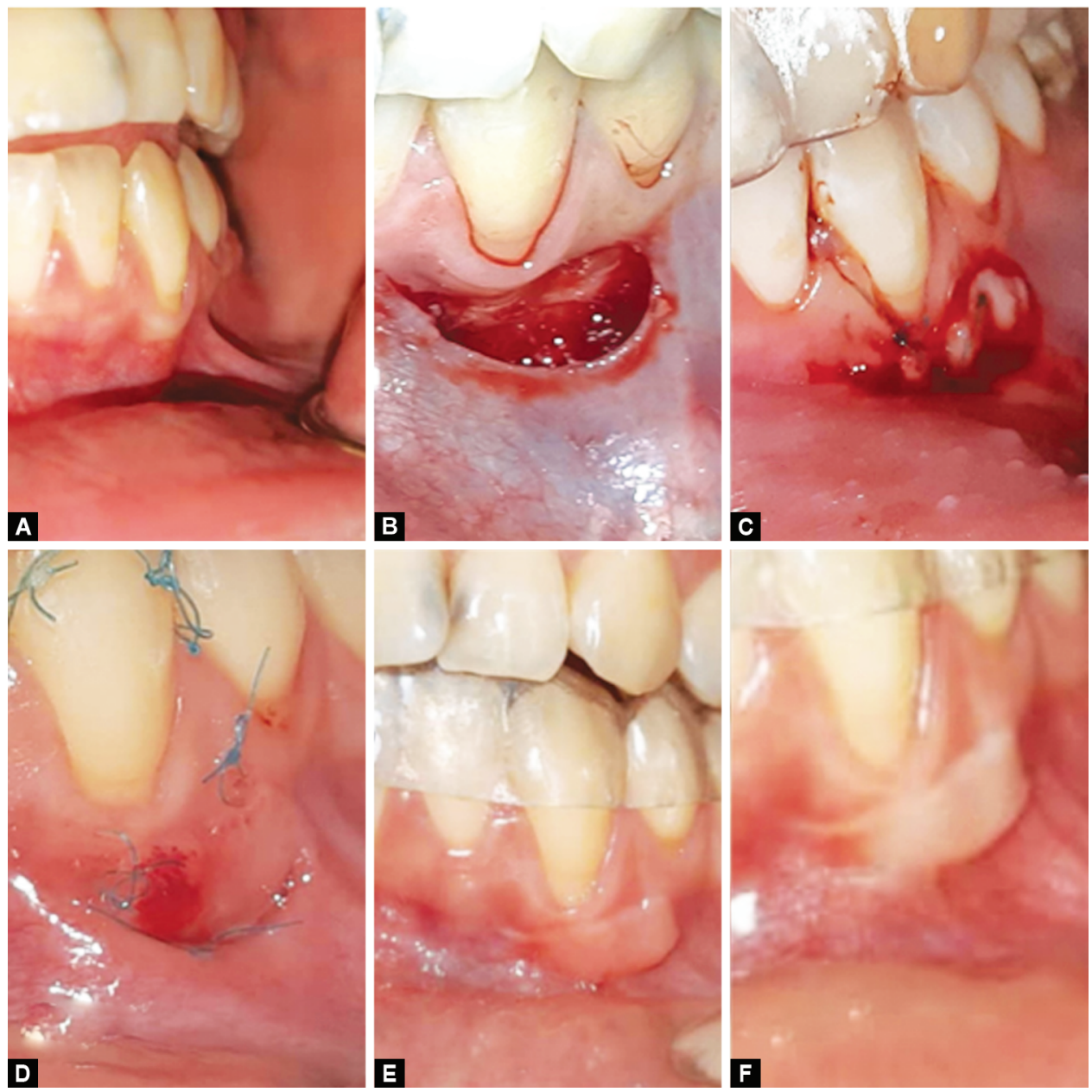

Figs 2A to F: Hyaluronic acid (HA) side: (A) Gingival recession Miller's class II on tooth 43; (B) Recipient bed prepared; (C) Adaptation of the free gingival graft at the recipient area, after HA application, suture tide; (D) Graft healing at 14th day; (E) 1 month; (F) 3 months post-surgery

placed over the recipient site until the graft was harvested from the palate. The graft dimensions were the same in both sides based on a customized aluminum foil for each patient.

The transplanted graft was adapted to the recipient site with gentle compress and sutured with 6-0 nylon sutures.

The palatal wound was covered by a periodontal dressing and an acrylic stent was placed on the donor site for 1 week to stabilize the dressing.

\section{Free Gingival Graft + Hyaluronic Acid Group (Test Group)}

Same surgical steps were followed as in the control group but HA gel (Klipdent, Valdmiva, Russia) was applied to the recipient site using sterile syringe containing $1 \mathrm{~mL}$ of the gel which has a slowdissolving pattern prior to graft suturing. Patients were blinded of which side received the gel (Fig. 3).

\section{Free Gingival Graft Group (Control Group)}

Free gingival graft was performed without any additives.

\section{Postoperative Care}

Acetaminophen $500 \mathrm{mg}$ was prescribed four tablets/day for 3 days. Patients were instructed not to use dental brush in the surgical area until sutures removal after 2 weeks. A $0.12 \%$ chlorhexidine rinse three times daily was prescribed in order to inhibit plaque accumulation.

\section{Clinical Parameters}

Wound Healing Index (Landry, Turnbull, Howley index): $:^{20}$

Healing was evaluated with the following parameters: Erythema, bleeding, and granulation tissues, and was estimated in days $3,7,14,21$, and 28 with a 5-level score index: Grade (1) very poor healing and grade (5) excellent healing.

\section{Swelling Index}

Grade (0): No swelling or inflammation. Grade (1): Mild, intraoral swelling localized to the surgical region. Grade (2): Moderate, extraoral swelling localized to the surgical region. Grade (3): Marked, extraoral swelling spreading beyond the surgical region. Grade (4): Severe, extraoral swelling spreading beyond the surgical region and limited mouth opening.

\section{Postoperative Pain}

A visual analog scale (VAS) graded from 0 to 10 was used by the patient to record postoperative pain intensity daily for the first week. Grade (0) is no pain and grade (10) is extreme pain. Patients were also asked to record the number of analgesics intake. 

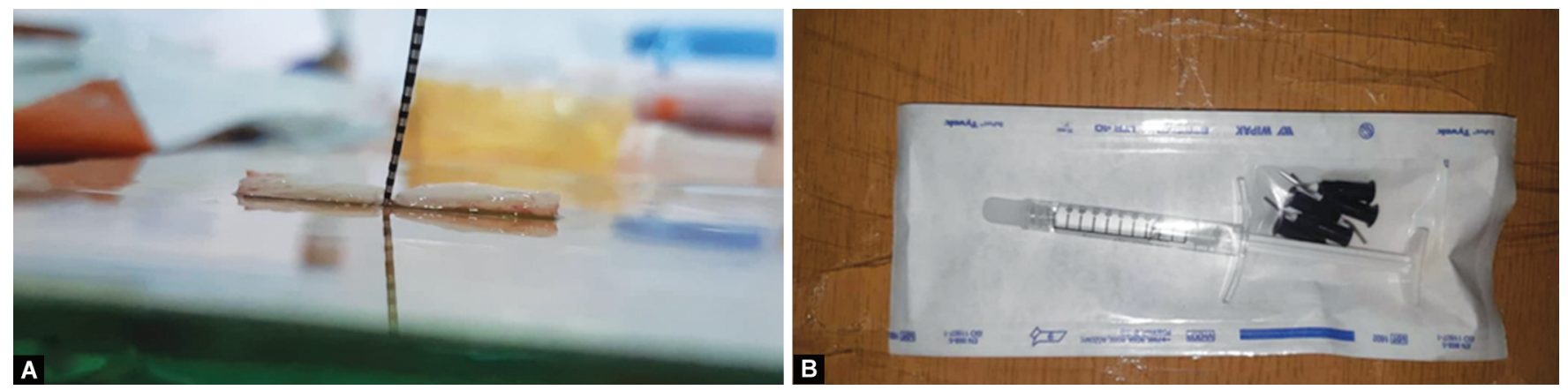

Figs $3 \mathrm{~A}$ and $\mathrm{B}$ : (A) Harvested grafts thickness; (B) A sterile syringe containing hyaluronic acid gel

Table 1: Comparison of the healing index in two groups between the periods study

\begin{tabular}{lllll}
\hline & Day & Group & Mean & p value \\
\hline Wound healing index & 3rd day & Control & $1.73 \pm 0.45$ & 0.235 \\
& & Test & 1.64 & \\
& 7th day & Control & $2.73 \pm 0.45$ & $\mathbf{0 . 0 3 5}$ \\
& & Test & 3 & \\
& 14th day & Control & 3 & $\mathbf{0 . 0 4 3}$ \\
& Test & $3.9 \pm 0.41$ & \\
& 21st day & Control & 3.9 & 0.164 \\
& Test & $4.06 \pm 0.25$ & \\
& 28th day & Control & $4.26 \pm 0.45$ & 0.104 \\
& & Test & $4.3 \pm 0.41$ & \\
\hline
\end{tabular}

*Mann-Whitney $U$

Bold term indicate $p$ value is statistically significant

\section{Graft Dimensional Changes Evaluation}

A headgear with a special arm to hold the camera in specific repeatable manner was designed and photographs of the surgical sites were taken in order to ensure that we had the same reference position during the follow-up period. The photographs were analyzed by the examiner who was blinded about the treatment protocol; a computer-based analysis program (Image National Institutes of Health, USA) was used. The photographs were taken at baseline and at 1-and 3-month follow-up.

\section{Statistical Analysis}

Statistical testing was carried out using SPSS (version 21, Chicago, Illinois, USA). A descriptive analysis was conducted (standard deviation, mean, and frequency distribution) for the collected data. The Wilcoxon test was used to analyze the differences over time in the same group. The Mann-Whitney U-test was used to assess intragroup comparisons. Data were considered statistically significant at level $<0.05$.

\section{Results}

Of 18 participant patients first treated, 3 did not complete the treatment or the follow-ups. Fifteen patients ( 12 females, 3 males) completed the follow-up period.

\section{Healing, Edema, and Postoperative Pain}

The findings demonstrated statically significant differences for graft healing values between the two groups at days 7 and 14 ( $p=0.035$, $p=0.045)$, respectively. However, no significant differences were determined at days 21 and 28 (Table 1). Whereas swelling was
Table 2: Patient morbidity at 7 days postsurgical days (VAS)

\begin{tabular}{llll}
\hline Parameters & Test & Control & $p$ value \\
\hline Pain & $0(1)$ & $1(1)$ & 0.351 \\
Swelling & $1(1)$ & $2(1)$ & $\mathbf{0 . 0 3 5}$ \\
\hline
\end{tabular}

*Mann-Whitney $U$

Bold term indicate $p$ value is statistically significant

statistically significant lower in the HA group after 7 days ( $p=0.021)$, no such difference was found regarding pain intensity $(p=0.21)$ (Table 2).

\section{Photographic Analysis of the Graft Dimensions and Shrinkage}

Graft's dimensions data, which are summarized in Table 3, mesiodistal dimension 1st month post-surgery was $11.6 \pm 1.8$ $\mathrm{mm}$ and $11 \pm 1.5 \mathrm{~mm}$ in the test group and the control group, respectively, and the differences were not statistically significant ( $p$ $=0.275$ ). At 3 months post-surgery, results were also not statistically significant ( $p=0.31$ ) as in the test group was $10.9 \pm 1.8 \mathrm{~mm}$ while in the control group was $10.4 \pm 1.8 \mathrm{~mm}$. In the coronal-apical dimension, there were no statistical differences between the test and the control groups at 1 month post-surgery $(p=0.053)$ and 3 months post-surgery $(p=0.059)$. Table 3 shows that there is no statistical differences comparing graft area at 1 month post-surgery (test group $=96.7 \pm 18.5 \mathrm{~mm}^{2}$, control group $=84.2 \pm 14.7 \mathrm{~mm}^{2}$, and $p=0.052$ ) and after 3 months post-surgery (test group $=80.3$ $\pm 15 \mathrm{~mm}^{2}$, control group $=69.6 \pm 13.4 \mathrm{~mm}^{2}, p=0.058$ ).

Eventually by analyzing the results, it is clear that the FGG healing index is better in the test group and statistically significant at days 7 and 14 of following up and the edema index results were statistically significant in the test group and better from the control group and there is no difference in pain feeling after the surgery. Changes in gingival graft's dimensions and shrinkage post-surgery were less after 1 month and 3 months in the test group compared to the control group but statistically not significant.

\section{Discussion}

Many researches have studied the FGG healing, postoperative complications, and dimensional changes.

In this split-mouth, clinical study two FGGs were conducted in the mandibular canine region at the same session. Both grafts had similar dimensions. The attached gingiva width was $<2 \mathrm{~mm}$ with class I or II Miller GR.

On the test side, HA gel was used prior to graft placement and gentile compress was applied to the graft on the recipient site. On the control side, no gel was applied to the recipient site. The grafts were sutured with 6-0 nylon sutures. 
Table 3: Mean values and standard deviations of the transplanted tissue in horizontal and vertical dimensions including graft areas

\begin{tabular}{|c|c|c|c|c|}
\hline & & Baseline & First month & Third month \\
\hline \multirow[t]{9}{*}{ Mesiodistal sizes (horizontal) (mm) } & Test & & & \\
\hline & Mean $\pm S D$ & $12.5 \pm 1.6$ & $11.6 \pm 1.8$ & $10.9 \pm 1.8$ \\
\hline & Min-Max & $10-15$ & $9-15$ & $9-14$ \\
\hline & Med & 12 & 11 & 10 \\
\hline & Control & & & \\
\hline & Mean \pm SD & $12.5 \pm 1.6$ & $11 \pm 1.5$ & $10.4 \pm 1.5$ \\
\hline & Min-Max & $10-15$ & $9-14$ & $8-13$ \\
\hline & Med & 12 & 11 & 10 \\
\hline & $p$ value & 1 & 0.275 & 0.31 \\
\hline \multirow[t]{9}{*}{ Apical-coronal sizes (vertical) (mm) } & Test & & & \\
\hline & Mean $\pm S D$ & $9.6 \pm 0.8$ & $8.3 \pm 0.8$ & $7.4 \pm 0.8$ \\
\hline & Min-Max & $8-11$ & $7-10$ & $6-9$ \\
\hline & Med & 10 & 8 & 7 \\
\hline & Control & & & \\
\hline & Mean \pm SD & $9.6 \pm 0.8$ & $7.7 \pm 0.7$ & $6.9 \pm 0.5$ \\
\hline & Min-Max & $8-11$ & $6-9$ & $6-8$ \\
\hline & Med & 10 & 8 & 7 \\
\hline & $p$ value & 1 & 0.053 & 0.059 \\
\hline \multirow[t]{9}{*}{ Graft area $\left(\mathrm{mm}^{2}\right)$} & Test & & & \\
\hline & Mean $\pm S D$ & $121.5 \pm 22.2$ & $96.7 \pm 18.5$ & $80.3 \pm 15$ \\
\hline & Min-Max & $96-165$ & $77-140$ & $60-126$ \\
\hline & Med & 120 & 90 & 80 \\
\hline & Control & & & \\
\hline & Mean \pm SD & $182.3 \pm 22.2$ & $84.2 \pm 14.7$ & $69.6 \pm 13.4$ \\
\hline & Min-Max & $96-165$ & $66-126$ & $56-84$ \\
\hline & Med & 120 & 80 & 63 \\
\hline & $p$ value & 1 & 0.052 & 0.058 \\
\hline
\end{tabular}

SD, Standard deviation; Min, minimum; Max, maximum

*Mann-Whitney U

During the 3-month follow-up, the clinical findings demonstrated statically significant better graft healing scores in day $7(p=0.035)$ and day $14(p=0.045)$ in the test group (Figs 1D and 2D).

The accelerated healing process can be referred to the antiinflammatory role of the HA through its physical characteristics which provide stability and flexibility to the tissues and delay the microbial penetration. ${ }^{21,22}$ The HA aids in wound closure and accelerates the healing process by interfering with the interactions between the inflammatory cells and its ability to scavenge the inflammatory cell-derived reactive oxygen species. ${ }^{23}$

Furthermore, this study demonstrated statistically significant differences between the test and the control groups during the first postoperative week in swelling. This result can be referred to the HA moisturizing properties and its effectiveness in accelerating migration of the lymphocytes and inflammatory cells in the wound and activation of the connective tissue cells. ${ }^{24}$

In Pilloni et al. study which compared between the coronally advanced flap (CAF) alone and CAF with topical HA and found that HA application reduces the post-surgery edema where $p=0.01$ while no difference were noticed in the pain as $p=0.151{ }^{25}$

The systematic review of Casale et al. analyzed the statistical results of 20 studies for topical application of $\mathrm{HA}$ and its effect on gingival inflammation, chronic periodontitis, and implantation, sinus lifting and oral ulcers. This systematic review showed that the topical application of HA accelerates tissues and wounds healing in the oral cavity and participated in gingival inflammation healing and chronic periodontitis healing as well as improving patient's life quality. ${ }^{21}$

Romeo et al. study about amino acid and hyaluronate sodium impact on oral wound healing after using the laser for biopsy showed a statistically significant difference in tissue healing index for the hyaluronic, while there were no differences in pain index. ${ }^{26}$

In our study, no statistically significant differences were found for pain values during all 7 days following the surgery between both groups. This can be explained by the individual variations in pain threshold.

In previous studies, graft dimensional changes were evaluated by measuring the vertical and the horizontal dimensions using a periodontal probe, a digital caliber, or a computer-based photograph analysis., $8,27,28$

Hatipo and Keçeli studied the dimensional changes of the FGG at 10,21, and 180 days. No horizontal changes were observed during the study periods $(p>0.05)$. However, there were significant differences in the vertical dimension $(p<0.05){ }^{8}$

In our study, topical application of the HA in the recipient site reduced the graft shrinkage at 1- and 3-month periods. However, the differences were not statically significant between the groups (Table 3). 
The HA enhanced the graft blood supply through accelerating the neovascularization between the graft and the adjacent tissues leading to reduction in graft shrinkage and therefore, fewer abnormalities in the graft appearance. ${ }^{23}$

Çankaya et al. used HA in the recipient site and then applied laser Doppler flowmetry (LDF) laser to evaluate the graft blood flow. They found statically significant differences in vertical shrinkage of the graft at 1 month after surgery. ${ }^{29}$ We attribute the discrepancies with our study to the fact that they used the FGG procedure to deepen the vestibule in edentulous regions, thus providing more blood supply to the graft, whereas, our study included teeth in the surgical area which will impair the blood supply. Moreover, the soft tissue biotype and the individual variations in the healing process play a role in this disagreement.

This study has some methodological limitations, as we did not distinguish between gingival phenotypes before surgery, so for the future studies it is better to subdivide the groups based on the gingival phenotype to further evaluate the HA effect on graft healing and shrinkage. In addition, to a longer follow-up period.

\section{Conclusion}

The adjunctive use of topical HA with the FGG is an effective and safe method. The HA accelerates the graft healing and reduces the postoperative complications and side effects.

\section{References}

1. Armitage GC. Development of a classification system for periodontal diseases and conditions. Ann Periodontol 1999;4(1):1-6. DOI: 10.1902/ annals.1999.4.1.1.

2. Lang NP, Löe H. The relationship between the width of keratinized gingiva and gingival health. J Periodontol 1972;43(10):623-627. DOI: 10.1902/jop.1972.43.10.623.

3. Camargo PM, Melnick PR, Kenney EB. The use of free gingival grafts for aesthetic purposes. Periodontol 2000 2001;27(1):72-96. DOl: 10.1034/j.1600-0757.2001.027001072.x.

4. Chambrone L, Tatakis DN. Periodontal soft tissue root coverage procedures: a systematic review from the AAP regeneration workshop. J Periodontol 2015;86(2-s):S8-S51. DOI: 10.1902/ jop.2015.130674.

5. Zuhr O, Bäumer D, Hürzeler M. The addition of soft tissue replacement grafts in plastic periodontal and implant surgery: Critical elements in design and execution. J Clin Periodontol 2014;41(Suppl s):S123-S142. DOI: $10.1111 /$ jcpe.12185.

6. Bertl K, Melchard M, Pandis N, et al. Soft tissue substitutes in non-root coverage procedures: a systematic review and meta-analysis. Clin Oral Investig 2017;21(2):505-518. DOI: 10.1007/s00784-016-2044-4.

7. Mörmann W, Schaer F, Firestone AR. The relationship between success of free gingival grafts and transplant thickness: revascularization and shrinkage-a one year clinical study. J Periodontol 1981;52(2):74-80. DOI: 10.1902/jop.1981.52.2.74.

8. Hatipo $\mathrm{H}$, Keçeli HG. Vertical and horizontal dimensional evaluation of free gingival grafts in the anterior mandible: a case report series. 2007;11(2):107-113. DOI: 10.1007/s00784-006-0084-x.

9. Bartold PM, Wiebkin OW, Thonard JC, et al. Glycosaminoglycans of human gingival epithelium and connective tissue. 2016;9(2):99-106. DOI: 10.3109/03008208109160247.

10. Dahiya P, Kamal R. Hyaluronic acid: A boon in periodontal therapy. 2013;5(5):309-315. DOI: 10.4103/1947-2714.112473.

11. Fraser JRE, Laurent TC, Laurent UBG. Hyaluronan: its nature, distribution, functions and turnover. J Int Med 1997;242(1):27-33. DOI: 10.1046/j.1365-2796.1997.00170.x.

12. Weigelt PH, Fuller GM, Leboeuf RD. A Model for the Role of Hyaluronic Acid and Fibrin in the Early Events during the Inflammatory Response and Wound Healing Department of Human Biological Chemistry and Genetics, University of Texas. 1986.

13. Mendes RM, Silva GAB, Lima MF, et al. Sodium hyaluronate accelerates the healing process in tooth sockets of rats. 2008;53:1155-1162. DOI: 10.1016/j.archoralbio.2008.07.001.

14. Koray M, Ofluoglu D, Onal EA, et al. Efficacy of hyaluronic acid spray on swelling, pain, and trismus after surgical extraction of impacted mandibular third molars. Int J Oral Maxillofac Surg 2014;43(11):13991403. DOI: 10.1016/j.ijom.2014.05.003.

15. Bjørnland T, Gjærum AA, Møystad A. Osteoarthritis of the temporomandibular joint: an evaluation of the effects and complications of corticosteroid injection compared with injection with sodium hyaluronate. J Oral Rehabil, 2007;34(8):583-589. DOI: 10.1111/j.1365-2842.2007.01759.x.

16. Göçmen G, Atalı O, Aktop S. , Hyaluronic acid versus ultrasonic resorbable pin fixation for space maintenance in non-grafted sinus lifting. J Oral Maxillofac Surg 2016;74(3):497-504. DOI: 10.1016/j. joms.2015.10.024.

17. Nolan A, Baillie C, Badminton J, et al., PDFlib PLOP: PDF Linearization, Optimization, Protection Page inserted by evaluation version The efficacy of topical hyaluronic acid in the management of recurrent aphthous ulceration.

18. Xu Y, Höfling K, Fimmers R, et al. Clinical and microbiological effects of topical subgingival application of hyaluronic acid gel adjunctive to scaling and root planing in the treatment of chronic periodontitis. J Periodontol 2004;75(8):1114-1118. DOI: 10.1902/jop.2004.75.8.1114.

19. El-sayed KMF, Dahaba MA. Local application of hyaluronan gel in conjunction with periodontal surgery: a randomized controlled trial. Clin Oral Investig 2012;16(4):1229-1236. DOI: 10.1007/s00784011-0630-z.

20. Beausang E, Floyd H, Dunn KW, et al. A new quantitative scale for clinical scar assessment. Plast Reconstr Surg 1998;102(6):1954-1961. DOI: 10.1097/00006534-199811000-00022.

21. Casale M, Moffa A, Vella P, et al. Hyaluronic acid: perspectives in dentistry. A systematic review. Int J Immunopathol Pharmacol 2016;29(4):572-582. DOI: 10.1177/0394632016652906.

22. Yıldırım S, Özener HÖ, Doğan B, et al. Effect of topically-applied hyaluronic-acid on pain and palatal epithelial wound healing: an examiner-blind, randomized, controlled clinical trial. J Periodontol 2018;89(1):36-45. DOI: 10.1902/jop.2017.170105.

23. Noble PW. Hyaluronan and its catabolic products in tissue injury and repair. Matrix Biol 2002;21(1):25-29. DOI: 10.1016/S0945053X(01)00184-6.

24. Eick $S$, Renatus $A$, Heinicke $M$, et al. Hyaluronic acid as an adjunct after scaling and root planing: a prospective randomized clinical trial. J Periodontol 2013;84(7):941-949. DOI: 10.1902/jop.2012.120269.

25. Pilloni A, Schmidlin PR, Sahrmann P, et al. Correction to: effectiveness of adjunctive hyaluronic acid application in coronally advanced flap in miller class I single gingival recession sites: a randomized controlled clinical trial. Clin Oral Investig 2018;22(8):2961-2962. DOI: 10.1007/ s00784-018-2567-y.

26. Romeo U, Libotte F, Palaia G, et al. Oral soft tissue wound healing after laser surgery with or without a pool of amino acids and sodium hyaluronate: a randomized clinical study. Photomed Laser Surg 2014;32(1):10-16. DOI: 10.1089/pho.2013.3509.

27. Çifçibaşı E, Karabey V, Koyuncuoğlu C, et al. Clinical evaluation of free gingival graft shrinkage in horizontal and vertical dimensions. J Istanbul Univ Fac Dent 2015;49(3):11. DOI: 10.17096/jiufd.58759.

28. Yildiz MS, Gunpinar S. Free gingival graft adjunct with low-level laser therapy: a randomized placebo-controlled parallel group study. Clin Oral Investig 2019;23(4):1845-1854. DOI: 10.1007/s00784-0182608-6.

29. Çankaya Z, Gürbüz S, Bakirarar B, et al. Evaluation of the effect of hyaluronic acid application on the vascularization of free gingival graft for both donor and recipient sites with laser Doppler flowmetry: a randomized, examiner-blinded, controlled clinical trial. Int J Periodont Restorat Dent 2020;40(2):233-243. DOI: 10.11607/prd.4494. 